\title{
High-resolution Velocity Fields of Low-mass Disk Galaxies. I. CO Observations
}

\author{
Phuongmai N. Truong ${ }^{1}$, Andrew B. Newman ${ }^{2}$, Joshua D. Simon ${ }^{2}$, Leo Blitz ${ }^{3}$, Richard Ellis ${ }^{4}$, and Alberto Bolatto ${ }^{5}$ \\ ${ }^{1}$ Department of Physics, University of California, Berkeley, CA 94720, USA; mai.truong@ berkeley.edu \\ ${ }^{2}$ Carnegie Observatories, Pasadena, CA 91101, USA \\ ${ }^{3}$ Department of Astronomy, University of California, Berkeley, CA 94720, USA \\ ${ }^{4}$ European Southern Observatory, Munich, Germany \\ ${ }^{5}$ Department of Astronomy, University of Maryland, College Park, MD 20742, USA \\ Received 2016 November 17; revised 2017 May 30; accepted 2017 May 31; published 2017 June 29
}

\begin{abstract}
This paper is the first in a series whose aim is to examine the relative distributions of dark and baryonic matter as a function of star formation history in a representative sample of low-mass disk galaxies. In this paper, we present high-resolution ${ }^{12} \mathrm{CO}(J=1 \rightarrow 0)$ interferometry for a sample of 26 nearby dwarf galaxies that were obtained from the Combined Array for Research in Millimeter-wave Astronomy (CARMA). Among these 26 galaxies, 14 have good CO detections, including 6 galaxies previously detected in single-dish CO measurements and 8 newly detected ones. We find a linear correlation between the CO flux and the mid- and far-IR flux from the WISE and IRAS catalogs. Compared to the far-IR flux, the mid-IR flux may be a better indication of whether a galaxy contains sufficient $\mathrm{CO}$ for detection at the level of instrument sensitivity of CARMA. This correlation might prove to be useful in future studies to help choosing other $\mathrm{CO}$ targets for observation. The median molecular mass (including helium) of our galaxies is $2.8 \times 10^{8} M_{\odot}$, which is consistent with past observations for dwarf galaxies. The molecular content is weakly correlated with the dynamical mass, $r$-band luminosity and size of the galaxies. The median ratios of molecular mass versus dynamical mass and molecular mass versus $r$-band luminosity are $M_{\mathrm{mol}} / M_{\mathrm{dyn}} \approx 0.035$ and $M_{\mathrm{mol}} / L_{r} \approx 0.078 M_{\odot} / L_{r, \odot}$, respectively, which are also consistent with past observations for dwarf galaxies.
\end{abstract}

Key words: dark matter - galaxies: dwarf - galaxies: halos - galaxies: kinematics and dynamics - galaxies: spiral

\section{Introduction}

Cosmological simulations have shown that cold dark matter models $(\mathrm{CDM}$ and $\Lambda \mathrm{CDM})$ give rise to a universal density profile for dark matter (DM) halos: NFW profiles (Navarro et al. 1996), where the density increases with decreasing radius in the central region $\left(\rho \propto r^{-\alpha}\right.$, where $\alpha=1$ for $r$ smaller than the scale radius). In other words, these simulations suggest a central-cusp density profile for halos ranging from dwarf galaxies to galaxy clusters. However, observational studies of nearby dwarf galaxies have found density profiles in the central region spanning a wide range of distributions from constantdensity cores to nearly cuspy profiles (e.g., Borriello \& Salucci 2001; de Blok et al. 2001a; Simon et al. 2005; Oh et al. 2011; Adams et al. 2014). This inconsistency between simulations and observations at the inner parts of galaxies is known as the core-cusp problem in galactic-scale cosmology.

Over the past two decades, much theoretical work has been devoted to explaining the observed core-like DM distributions in some galaxies. The mechanisms to reduce the central density can be classified into three categories:

1. structural features in real galaxies, such as clumps of mass or bars. The relative motion of the features transfers energy or angular momentum from baryons to DM, pushes DM outward and flattens the initial cusp (e.g., El-Zant et al. 2001; Tonini et al. 2006; Weinberg \& Katz 2007a, 2007b). However, such features were not included in the $N$-body simulations of, e.g., Navarro et al. (1996);

2. non-CDM models (warm DM, self-interacting DM, dissipative DM, etc.; e.g., Kaplinghat 2005; Rocha et al. 2013; Foot 2016);
3. baryonic feedback, most notably the repeated expansion and contraction of the interstellar gas caused by supernovae (e.g., Pontzen \& Governato 2012; Arraki et al. 2014; Kato et al. 2016; Chan et al. 2015 etc.).

Although some of these simulations and models have been disputed (as explained in more detail in Adams et al. 2014), it is true that several theories exist to explain the shallow density profiles of dwarf galaxies. At larger masses, studies of galaxy clusters using kinematics and gravitational lensing to constrain the mass show that the inner regions $\left(\leqslant 30 \mathrm{kpc} \sim 0.003-0.03 r_{200}\right)$ have shallower density profiles than the NFW profiles (Newman et al. 2013a, 2013b). If the density profile measurements in the literature are accurate, it is unclear whether any single explanation can account for these observed profiles over many orders of magnitude in halo mass or whether several mechanisms must act together over different mass scales.

On the observational side, there are many analyses of $\mathrm{HI}$ data (de Blok et al. 1996; de Blok \& McGaugh 1997; Oh et al. 2011, 2015), optical long-slit spectroscopy data (McGaugh et al. 2001; van den Bosch \& Swaters 2001; de Blok et al. 2001a, 2001b; de Blok \& Bosma 2002; Swaters et al. 2003a), and integral field spectrograph (IFS) data (Simon et al. 2003, 2005; Swaters et al. 2003b; Kuzio de Naray et al. 2006; Adams et al. 2014) on the subject. These data have shown that late-type dwarfs generally have rotation curves that rise more slowly than the NFW profile, although the highestquality data do not prefer strongly cored profiles. However, the interpretation of long-slit spectra along the major axis of the galaxies could be subjected to various systematic errors, such as misalignment of the position angle of the slit with that of the galaxy, missing information on the asymmetries, or non-circular motions of the gas. IFU and interferometric maps 
are less susceptible to such systematic errors and can quantify non-circular motions over a range of azimuthal angles. However, improved resolution, sensitivity, and analysis techniques have not removed the core-like DM profiles in H I data, suggesting that they are unlikely to be manifestation of observational uncertainties alone (Oh et al. 2011). In recent years, three high-resolution surveys of dwarf galaxies have been undertaken to examine this core-cusp problem: 7 THINGS galaxies (Oh et al. 2011), 26 LITTLE THINGS galaxies (Oh et al. 2015; note that the subset of THINGS and LITTLE THINGS galaxies that is useful for detailed mass modeling is much smaller than the full samples), and another 7 dwarfs in Adams et al. (2014), who used SDSS photometric data, archival $\mathrm{H}$ I data, and IFU data from VIRUS-W. All three samples consistently show that the logarithmic inner slope of the DM density profiles with respect to radius is significantly lower than 1: the average value of $\alpha$ is $0.29 \pm 0.07$ (Oh et al. 2011), $0.32 \pm 0.24$ (Oh et al. 2015), and $0.58 \pm 0.24$ (Adams et al. 2014).

In an attempt to increase the sample size, obtain better constrained rotation curves, and resolve the long-standing discrepancy between simulations and observations, we have begun a new survey aimed at measuring the DM distribution in a large sample of dwarf galaxies using high-resolution $\mathrm{CO}$ interferometry and $\mathrm{H} \alpha$ IFU spectroscopy. On one hand, the $\mathrm{CO}$ measurements help us probe near the center of the galaxies to obtain the least biased inner slope of the rotation curves. On the other hand, the $\mathrm{H} \alpha$ measurements let us observe the rotation curve further out than it is possible with $\mathrm{CO}$, which enables us to better constrain the functional form of the rotation curves and consequently the DM distribution. In detail, the advantages of $\mathrm{CO}$ (and $\mathrm{HI}$ ) interferometry are:

1. improved spectral resolution: $\mathrm{CO}$ resolution is approximately $2.5 \mathrm{~km} \mathrm{~s}^{-1}$; $\mathrm{H}$ I resolution $\approx 2.3 \mathrm{~km} \mathrm{~s}^{-1}$ (Oh et al. 2008); IFU resolution $\approx 35 \mathrm{~km} \mathrm{~s}^{-1}$ (Adams et al. 2014);

2. unaffected by extinction;

3. most molecular cloud emission is concentrated at the center of the galaxies, which is the region of interest;

4. complete spectral imaging information to produce reliable rotation curves;

5. better tracer of the gravitational potential near the center than $\mathrm{H} \alpha$ (ionized gas is subjected to "champagne flows," which affects the $\mathrm{H} \alpha$-derived velocity curve, whereas $\mathrm{CO}$ emission is from giant molecular clouds and does not exhibit this flow behavior). In addition, at least part of the $\mathrm{H} \alpha$ emission may be in a thick disk in some galaxies, the upper layers of which will have lower rotation velocities.

The CO interferometric data were obtained at Combined Array for Research in Millimeter-wave Astronomy (CARMA). The $\mathrm{H} \alpha$ integral field spectroscopy data are obtained using the Cosmic Web Imager (Matuszewski et al. 2010) at Palomar Observatory (N. C. Relatores et al. 2017, in preparation). Together with the $\mathrm{CO}$ data, the $\mathrm{H} \alpha$ data give us a check on the systematic errors. From the rotation curves obtained, we intend to subtract the stellar mass contribution (measured from nearIR imaging data), $\mathrm{HI}$ mass contribution (from archival $\mathrm{HI}$ data), and $\mathrm{H}_{2}$ mass contribution (deduced from the obtained CO measurements) to get the DM component (e.g., Simon et al. 2005). The project, which we call the Dwarf Galaxy Dark Matter (DGDM) Survey, will measure the radial distribution of $\mathrm{DM}$ at the scale of $\sim 100 \mathrm{pc}$ and help correlate the DM properties with the baryonic mass, gas density, star formation history, age, and other properties of the galaxy sample.

In this paper, we present the $\mathrm{CO}$ data in the following order: Section 2 describes our criteria to select galaxies for the survey; Section 3 describes the CARMA observation; Section 4 describes the data reduction; Section 5 presents the reduction results and discussion, which includes the comparisons between our $\mathrm{CO}$ flux and past observations (single-dish $\mathrm{CO}$ measurements and infrared measurements) and correlations between the molecular gas content and atomic gas content; Section 6 is the conclusion. To examine the mass distribution and star formation history of the galaxies in the sample, the remainder of the series will contain $\mathrm{CO}$ rotation curves and inferred dark matter profiles, with a brief comparison of the $\mathrm{H} \alpha$ and $\mathrm{CO}$ rotation curves for a subset of the sample (P. N. Truong et al. 2017, in preparation), and a presentation of the full $\mathrm{H} \alpha$ sample, including the rotation curves, photometry, and mass modeling (N. C. Relatores et al. 2017, in preparation). By comparing and combining $\mathrm{CO}$ and $\mathrm{H} \alpha$ data, we will be able to draw conclusive results on the robustness of the DM slope, its distributions and scatter, and any correlations with galaxy properties.

\section{Target Selection}

In preparation for the DMDG survey, we compiled a list of 172 nearby $\mathrm{H}$ I-detected and $\mathrm{CO}$-detected small-mass galaxies from the literature ${ }^{6}$ using the following criteria.

1. The galaxies are low-mass galaxies, which we define as galaxies with an $\mathrm{H}$ I line width $\left(W_{20}\right) \leqslant 200 \mathrm{~km} \mathrm{~s}^{-1}$. As a matter of convenience, we will call them "dwarfs" henceforth, although traditional dwarf galaxies tend to have even lower masses and rotational velocities.

2. The galaxies have low luminosity, i.e., absolute magnitude $M_{B} \geqslant-18$.

3. The distance is less than $35 \mathrm{Mpc}$; the systemic velocity in the local standard of rest $\left(v_{\mathrm{LSR}}\right)$ is below $2000 \mathrm{~km} \mathrm{~s}^{-1}$. This limit provides better spatial resolution than $80 \mathrm{pc}$ for all targets.

Of these 172 galaxies, 63 have strong $\mathrm{CO}$ detections and 42 have published upper limits in the literature. For the remaining 67 galaxies, some have been observed and detected in ${ }^{12} \mathrm{CO}(1-0)$, but no numerical value of the $\mathrm{CO}$ flux or intensity was published.

Among these 172 galaxies, we further narrowed our preferences to select dwarfs that have the following properties (in addition to the above three criteria):

1. symmetrical, non-disturbed visual appearance

2. not being classified as a barred galaxy and not containing a visually obvious bar

3. inclination angle of $30^{\circ}-70^{\circ}$,

4. diameters $>1$ arcmin (to provide enough extent for the $\mathrm{H} \alpha$ measurements).

5. strong mid-IR flux. This criterion was selected using the infrared images provided by WISE (Wright et al. 2010).

\footnotetext{
The galaxies were selected from the following compilations: Leroy et al. (2005), Karachentsev et al. (2003), Walter et al. (2008; the THINGS catalog), Leroy et al. (2009; HERACLES), Ott et al. (2012; VLA ANGST), Mateo (1998), Young et al. (1995), Obreschkow \& Rawlings (2009), Welch \& Sage (2003), Matthews et al. (2005), Sauty et al. (2003), Andreani et al. (1995), Sage (1993), Lees et al. (1991), and Swaters et al. (2002).
} 
Table 1

List of Dwarf Galaxies with CO Observations from CARMA

\begin{tabular}{|c|c|c|c|c|c|c|c|c|c|c|c|c|}
\hline Name & R.A. & Decl. & $\begin{array}{c}v_{\mathrm{rot}} \\
\left(\mathrm{km} \mathrm{s}^{-1}\right)\end{array}$ & $\begin{array}{c}v_{\mathrm{LSR}} \\
\left(\mathrm{km} \mathrm{s}^{-1}\right)\end{array}$ & $\begin{array}{c}\text { Distance } \\
(\mathrm{Mpc})\end{array}$ & $M_{B}$ & Type & $\begin{array}{l}\text { Diameter } \\
\text { (arcmin) }\end{array}$ & $\begin{array}{l}\text { Diameter } \\
(\mathrm{kpc})\end{array}$ & $\begin{array}{l}\text { Inclination } \\
\text { (deg) }\end{array}$ & $\begin{array}{c}\text { PA } \\
(\operatorname{deg})\end{array}$ & Source \\
\hline NGC 746 & $01: 57: 51.0$ & $44: 55: 07$ & 58 & 704 & 12 & -16.9 & $\mathrm{Im}$ & 1.9 & 6.63 & 51 & 90 & HL \\
\hline NGC 853 & $02: 11: 41.2$ & $-09: 18: 22$ & 60 & 1520 & 20 & -16.2 & $\mathrm{Sm}$ & 1.5 & 8.72 & 56 & 65 & $\mathrm{HL}$ \\
\hline NGC 949 & 02:30:48.6 & $37: 08: 12$ & 91 & 607 & 10 & -17.8 & $\mathrm{Sab}$ & 2.4 & 6.81 & 68 & 145 & L05 \\
\hline NGC 959 & $02: 32: 23.9$ & $35: 29: 41$ & 74 & 594 & 10 & -17.2 & $\mathrm{Sdm}$ & 2.3 & 6.69 & 62 & 70 & L05 \\
\hline NGC 1012 & 02:39:14.9 & 30:09:05 & 96 & 982 & 18 & -18.6 & S0/a & 2.5 & 13.09 & 68 & 20 & L05 \\
\hline NGC 1035 & $02: 39: 29.1$ & 8:07:59 & 116 & 1229 & 17 & -18.3 & $\mathrm{Sc}$ & 2.5 & 12.36 & 75 & 150 & HL \\
\hline UGC 3371 & $05: 56: 38.6$ & $75: 18: 58$ & 72 & 821 & 18 & -17 & $\mathrm{Im}$ & 4.6 & 24.09 & 46 & 130 & L05; S02 \\
\hline UGC 4169 & 08:02:32.9 & $61: 23: 17$ & 107 & 1608 & 32 & -19.2 & Scd & 1.5 & 13.96 & 63 & 110 & $\mathrm{HL}$ \\
\hline NGC 3622 & $11: 20: 12.4$ & $67: 14: 30$ & 73 & 1305 & 27 & -18.9 & $\mathrm{Sc}$ & 1.3 & 10.36 & 68 & 50 & $\mathrm{HL}$ \\
\hline NGC 4150 & $12: 10: 33.6$ & $30: 24: 06$ & $\ldots$ & 225 & 14 & -18.3 & So & 2.3 & 9.37 & 58 & 145 & L05 \\
\hline NGC 4310 & $12: 22: 26.3$ & $29: 12: 29.8$ & 79 & 919 & 10 & -17.4 & $\mathrm{SAB}$ & 2.2 & 6.21 & 57 & 160 & L05 \\
\hline NGC 4376 & $12: 25: 18.0$ & $5: 44: 28$ & 73 & 1139 & 14 & -16.8 & I & 1.4 & 5.70 & 62 & 70 & HL \\
\hline NGC 4396 & $12: 25: 58.8$ & $15: 40: 17$ & 88 & -124 & 16 & $\ldots$ & Scd & 3.3 & 15.17 & 72 & 120 & L05 \\
\hline NGC 4451 & $12: 28: 40.5$ & $9: 15: 32$ & 116 & 868 & 27 & -17.7 & $\mathrm{Sbc}$ & 1.5 & 11.78 & 54 & 160 & HL \\
\hline NGC 4632 & $12: 42: 32$ & $-00: 04: 57$ & 108 & 1726 & 18.5 & -20.6 & $\mathrm{Sc}$ & 3.1 & 16.68 & 71 & 62 & HL \\
\hline NGC 4701 & $12: 49: 11.6$ & $3: 23: 19$ & 101 & 725 & 17 & -18.4 & $\mathrm{Sc}$ & 2.8 & 13.85 & 43 & 50 & L05 \\
\hline NGC 5204 & $13: 29: 36.5$ & $58: 25: 07$ & 56 & 213 & 5 & -16.6 & $\mathrm{Sm}$ & 5.0 & 7.27 & 59 & 172 & L05; S02 \\
\hline UGC 8516 & $13: 31: 52.6$ & 20:00:04 & 60 & 1033 & 23 & -17.6 & Scd & 1.1 & 7.44 & 49 & 45 & HL \\
\hline NGC 5303 & $13: 47: 45.0$ & $38: 18: 17$ & 83 & 1431 & 24 & -18.5 & $\mathrm{Sbc}$ & 1.1 & 7.68 & 62 & 84 & HL \\
\hline NGC 5692 & $14: 38: 18.1$ & $3: 24: 37$ & 96 & 1573 & 30 & -19.02 & $\mathrm{~S}$ & 1.0 & 8.64 & 53 & 32 & $\mathrm{HL}$ \\
\hline NGC 5949 & $15: 28: 00.7$ & $64: 45: 48$ & 87 & 445 & 15 & -18.4 & $\mathrm{Sbc}$ & 2.4 & 10.47 & 69 & 143 & L05 \\
\hline NGC 6106 & $16: 18: 47.1$ & $07: 24: 39$ & 134 & 1464 & 25 & -19.2 & $\mathrm{Sc}$ & 2.5 & 18.18 & 59 & 145 & HL \\
\hline NGC 6207 & $16: 43: 03.7$ & $36: 49: 57$ & 115 & 871 & 20 & -19.4 & $\mathrm{Sc}$ & 3.0 & 17.45 & 65 & 20 & HL \\
\hline UGC 11891 & $22: 03: 33.9$ & $43: 44: 57$ & 87 & 508 & 9 & -16.4 & $\mathrm{Im}$ & 4.2 & 10.99 & 43 & 113 & HL \\
\hline UGC 12009 & $22: 22: 40.2$ & $37: 58: 39$ & 98 & 1252 & 20 & -18 & $\mathrm{Sb}$ & 1.4 & 8.14 & 60 & 175 & HL \\
\hline NGC 7320 & $22: 36: 03.4$ & $33: 56: 53$ & 82 & 797 & 19 & -18.4 & $\mathrm{Sd}$ & 2.2 & 12.16 & 68 & 109 & HL \\
\hline
\end{tabular}

Note. The galaxies were selected based on information in the following catalogs: HyperLEDA (denoted as "HL" in the "source" column), Leroy et al. 2005 (L05), and Swaters et al. 2002 (S02). The R.A., decl., $v_{\mathrm{LSR}}$, distance, type, and diameter (in arcminute) were obtained from the NASA/IPAC Extragalactic Database (NED) at http://ned.ipac.caltech.edu. (For the distance, we used the mean distance value from NED.) $v_{\text {rot }}, M_{B}$, inclination, and PA were obtained from the HyperLEDA catalog at http://www-obs.univ-lyon1.fr, with the exception of UGC 11891: the PA was not available in HyperLEDA and was obtained from our photometric measurements (N. C. Relatores et al. 2017, in preparation).

To show clear dust properties, the galaxies should be detectable in the infrared at $22 \mu \mathrm{m}$. Because there is a strong correlation between the $22 \mu \mathrm{m}$ flux and star formation rate, and consequently $\mathrm{CO}$, selecting galaxies with strong $22 \mu \mathrm{m}$ detection should maximize the chances of observing $\mathrm{CO}$ emission.

We also include 16 galaxies from the HyperLEDA catalog ${ }^{7}$ that satisfy these conditions. The heterogeneous nature and quality of HyperLEDA data generally do not affect the selection of galaxies in our sample because we verified the galaxy properties with the data in NED and WISE. The final list of selected targets is shown in Table 1. Distributions of several key properties of the target galaxies are displayed in Figure 1. The samples consist of mostly late-type spiral galaxies (Sbc-Sm) with optical linear diameters of $10-15 \mathrm{kpc}$ and typical dynamical masses $M_{\mathrm{dyn}}=v_{\mathrm{rot}}^{2} R / G \approx 10^{10} M_{\odot}$, where $v_{\text {rot }}$ is the maximum inclination-corrected rotational velocity obtained from HyperLEDA, and $R$ is the linear diameter.

\section{Observations}

To ensure both sufficient resolution and $\mathrm{S} / \mathrm{N}$, the galaxies were each observed in the $\mathrm{C}, \mathrm{D}$, and $\mathrm{E}$ configurations of CARMA. The array consisted of nine $6.1 \mathrm{~m}$ diameter

\footnotetext{
7 The HyperLEDA catalog is on the web at http://www-obs.univ-lyon1.fr.
}

telescopes and six $10.4 \mathrm{~m}$ diameter telescopes with singlepolarization SIS receivers for the $3 \mathrm{~mm}$ band. All observations were set up for the ${ }^{12} \mathrm{CO}(J=1 \rightarrow 0)$ spectral line (rest frequency: $115.271 \mathrm{GHz}$ ) and were single-pointing observations. The CARMA primary beam has a half-power diameter of $\sim 2$ arcmin. Three bands, each of which had a width of $62 \mathrm{MHz}$, 255 channels, and a channel width of $\sim 0.65 \mathrm{~km} \mathrm{~s}^{-1}$ $(\sim 0.25 \mathrm{MHz})$, were slightly overlapped to cover the entire signal. The combined velocity extent of the three bands was $410 \mathrm{~km} \mathrm{~s}^{-1}$.

The gain and phase of the array were calibrated by observing a nearby quasar for 3 minutes every 15 minutes. The quasar typically had a spectral flux density of $\sim 1 \mathrm{Jy}$ to provide sufficiently good calibration for our observations with three $62 \mathrm{MHz}$ bands. Systemic calibration errors were minimized by selecting a quasar close to the target galaxy (typically within $20^{\circ}$ ). The flux density scale $(\mathrm{Jy} / \mathrm{K})$ was determined from planetary observations (Mars, Uranus, Neptune) or observation of a bright source (e.g., MWC 349). The history of calibrator fluxes was maintained in the MIRIAD reduction software package. The calibrators were automatically determined by the CARMA observing tools when observing scripts were prepared using the CARMA system. Data collection for the project began in 2013 April and finished in 2015 March with over $600 \mathrm{hr}$ at CARMA. Table 2 shows the integration time in each array configuration for the galaxies. 

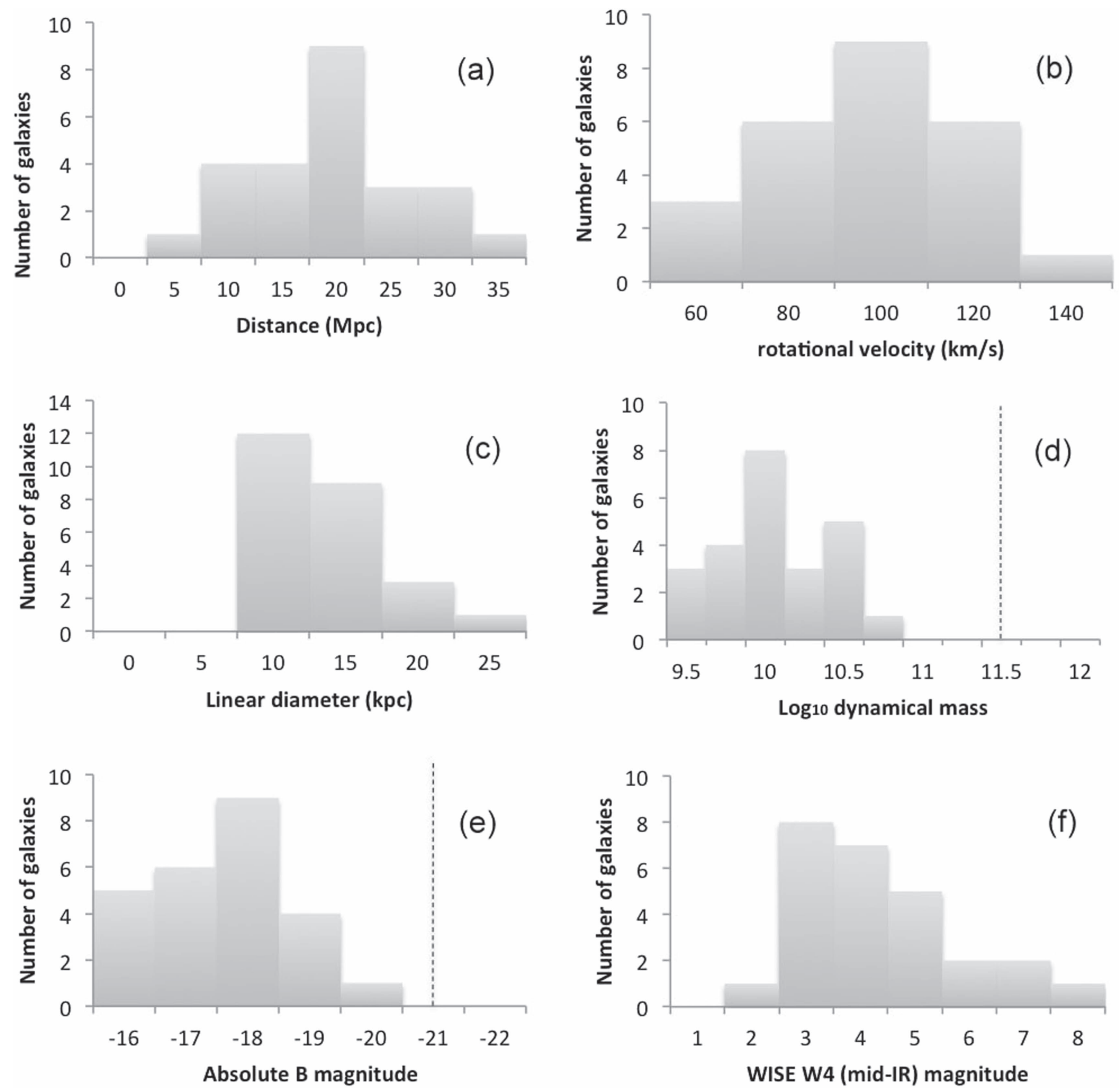

Figure 1. Summary of various properties of the galaxies targeted with CARMA in our survey: (a) distance (measured in Mpc); (b) rotational velocity (km $\mathrm{s}^{-1}$ ); (c) optical linear diameter (kpc), using the distances from (a) and major diameters ( $\operatorname{arcmin})$ from NED; (d) $\log _{10}$ of the dynamical mass $\left(M_{\odot}\right), M_{\mathrm{dyn}}=v_{\mathrm{rot}}^{2} R / G M_{\odot}$, with $v_{\text {rot }}$ from (b); (e) absolute blue magnitude; (f) WISE w4 mid-infrared magnitude. The black dotted lines in (d) and (e) indicate the approximate values for the Milky Way.

\section{Data Reduction}

To reduce the CARMA data, we used the INVERT, CLEAN, and RESTOR algorithms in the MIRIAD reduction package. In short, INVERT generates a spectral line cube from the visibilities; CLEAN deconvolves the dirty (synthesized) cube and dirty (synthesized) beam to produce a model of the deconvolved cube, which has only the clean components; the output of CLEAN is then used by RESTOR to produce a clean map of the observed target. For each target, the data were obtained in several data sets, which were calibrated individually; then, different data sets of the same target were reduced together to produce the final intensity (moment- 0 ) and velocity (moment-1) maps. The resulting naturally weighted maps had average synthesized beams of $\sim 3$ ". $5 \times 3^{\prime \prime}$, which corresponds to a physical resolution of $339 \times 291 \mathrm{pc}$ at a distance of $20 \mathrm{Mpc}$. The input visibility data sets had an average rms of $\sim 2.6 \mathrm{mJy}$ beam $^{-1}$ and a velocity resolution of $\sim 0.65 \mathrm{~km} \mathrm{~s}^{-1}$. Using the INVERT algorithm, the input spectral channels in the uv data sets are resampled at equal increments of $10 \mathrm{~km} \mathrm{~s}^{-1}$ with their weighted average to produce the image data cube. Negative-value pixels were clipped and masking (using the IMMASK algorithm) was applied to reduce the noise in the moment maps and enhance the map clarity.

Furthermore, we used the Matlab code described by Bolatto et al. (2002) and Simon et al. (2003) to calculate the velocity and velocity uncertainties per pixel in the moment-1 map. Both velocity and velocity uncertainty are necessary data for the 
Table 2

Integration Time in Each CARMA Array Configuration of the Dwarf Galaxies

\begin{tabular}{|c|c|c|c|}
\hline Name & C Array (h) & D Array (h) & E Array (h) \\
\hline NGC 746 & 0 & 7.2 & 0 \\
\hline NGC 853 & 6.5 & 17.5 & 0 \\
\hline NGC 949 & 10.1 & 15.8 & 9.5 \\
\hline NGC 959 & 7.3 & 6.2 & 0 \\
\hline NGC 1012 & 6.5 & 18.0 & 0 \\
\hline NGC 1035 & 8.4 & 16.6 & 6.4 \\
\hline UGC 3371 & 0 & 3.5 & 0 \\
\hline UGC 4169 & 0 & 4.1 & 0 \\
\hline NGC 3622 & 11.0 & 3.2 & 0 \\
\hline NGC 4150 & 23.0 & 31.7 & 4 \\
\hline NGC 4310 & 16.0 & 6.5 & 15.0 \\
\hline NGC 4376 & 9.2 & 7.3 & 0 \\
\hline NGC 4396 & 10.5 & 8.6 & 3.0 \\
\hline NGC 4451 & 7.0 & 10.4 & 0 \\
\hline NGC 4632 & 12.5 & 18.4 & 0 \\
\hline NGC 4701 & 7.2 & 16.0 & 0 \\
\hline NGC 5204 & 7.4 & 32.3 & 0 \\
\hline UGC 8516 & 0 & 6.4 & 0 \\
\hline NGC 5303 & 5.8 & 15.7 & 0 \\
\hline NGC 5692 & 10.2 & 22.5 & 0 \\
\hline NGC 5949 & 6.8 & 17.8 & 0 \\
\hline NGC 6106 & 6.5 & 7.2 & 17.1 \\
\hline NGC 6207 & 11.3 & 29.3 & 0 \\
\hline UGC 11891 & 9.5 & 4.0 & 0 \\
\hline UGC 12009 & 7.7 & 24.7 & 0 \\
\hline NGC 7320 & 18.7 & 7.0 & 0 \\
\hline
\end{tabular}

rotation curve fitting programs, which we will apply in the second part of this study (P. N. Truong et al. 2017, in preparation). The velocity uncertainty was calculated from straightforward error propagation and is expressed as

$$
d v=\frac{\mathrm{rms}}{m 0} \times \sqrt{\sum_{i=1}^{n}\left(v_{i}-m 1\right)^{2}},
$$

where $n$ is the number of velocity channels in the data cube; $v_{i}$ is the value of each velocity channel; $m 0=\sum_{i=1}^{n} s_{i}$ and $m 1=\sum_{i=1}^{n}\left(v_{i} s_{i}\right) / m 0$ are the common moment 0 and moment 1 of the data cube, with $s_{i}$ being the intensity of each pixel at velocity channel $v_{i}$.

Stringent constraints based on the rms and velocity range of the signal were applied in the algorithm to mask the pixels that would contribute mostly noise. In the data cube, we only used pixels with intensity $>2.5 \times \mathrm{rms}$ to isolate the signal in the spectrum. The velocity range of the emission was typically within $\pm 100 \mathrm{~km} \mathrm{~s}^{-1}$ of $v_{\text {sys. }}$.

Figure 2 shows the CO integrated intensity (whose values are denoted by the white contours) and velocity gradient of the targets with good $\mathrm{CO}$ detections. Among the 26 observed targets, 14 were detected in our measurements, 6 of which were detected in a previous CO single-dish measurement by Leroy et al. (2005). Two of the 12 undetected galaxies in our sample were detected in Leroy et al. (NGC 959 and NGC 4396), which might be due to the conditions of our observations: bad weather conditions on the observing days for these two targets, which reduced the amount of usable data; few hours of observation time (after the preliminary run, we increased the observing time for targets with clear signal by reallocating the time from targets with low or no signal), etc. Figure 3 shows the velocity maps of the same targets after masking was applied, where the
CO distributions appear much clearer with less noise. Note that several targets appear to have patchy $\mathrm{CO}$ distributions: NGC 853 has a hole in the center; NGC 1012, NGC 4701, NGC 4632, NGC 5303, NGC 5692, and NGC 5949 have a continuous distribution for up to only $\sim 5^{\prime \prime}$ from the center; (NGC 853, NGC 949, NGC 4451, NGC 6106, and NGC 6207 have a patchy ring of $\mathrm{CO}$ ); NGC 4150 has a notably small $\mathrm{CO}$ extension of only $\sim 10^{\prime \prime}$ in radius compared to the others. In Figure 4, we show the CO contours overlaid on $r$-band optical images. All $r$-band imaging data were taken with SPICAM on the ARC $3.5 \mathrm{~m}$ telescope at Apache Point Observatory (APO) on the nights of 2014 March 5 and November 27 (N. C. Relatores et al. 2017, in preparation), with the exception of NGC 4150 and NGC 4310: for these two galaxies, we used the $r$-band images in the SDSS III archive.

Figure 5 shows the spectra of the detections at the central position. The heliocentric radial velocities derived from optical and radio (mostly $\mathrm{HI}$ ) measurements are shown with vertical dotted and solid lines, respectively (these velocities were obtained from the hyperLEDA catalog). The range of maximum rotation velocity corrected for inclination is shown as the shaded region; this value was taken from the hyperLEDA catalog, which used the H I $21 \mathrm{~cm}$ line width $W_{20}$ and $\mathrm{H} \alpha$ rotation curves when available. The kinematic local-standard-of-rest (LSR) velocity (from NED) is shown with the vertical dashed lines. The systemic velocity as determined by MIRIAD from the $\mathrm{CO}$ data (Figure 3) is also shown with the vertical dash-dotted lines. The spectra were obtained at only the central position in the images using the algorithm MBSPECT in MIRIAD. We note that NGC 853, which has a hole in the center, exhibits a noisy spectrum.

The signals of the other galaxies fall within the corresponding $\mathrm{H}$ I line widths, which fall within the velocity extent of our observing windows. There are two exceptions: (i) NGC 4150 had no recorded rotation velocity in the catalog; (ii) NGC 5949: its recorded optical systemic velocity is almost $150 \mathrm{~km} \mathrm{~s}^{-1}$ greater than its recorded radio systemic velocity in HyperLEDA. The HyperLEDA optical $v_{\text {sys }}$ is likely an erroneous value because: (1) our measured $v_{\text {sys }}$ of NGC 5949 is $412 \mathrm{~km} \mathrm{~s}^{-1}$ (Figure 3), which is close to the HyperLEDA radio $v_{\text {sys, }}$, and (2) for this galaxy, Adams et al. (2014) found a heliocentric $v_{\text {sys }}$ of $440 \mathrm{~km} \mathrm{~s}^{-1}$ from fitting optical spectroscopy data.

In addition, we show the integrated $\mathrm{CO}$ profile of the galaxies in Figure 6, where the flux per velocity channel was summed over the entire data cube, where the pixels with intensity $<2.5 \times \mathrm{rms}$ were masked. One exception is NGC 5303, whose map is too noisy (Figure 2) and the pixels with intensity $<2 \times$ rms were masked because too few pixels had intensity above $2.5 \times$ rms. Because of the high noise level versus patchy CO distribution, NGC 5303 does not have a clear signal in the spectrum, but both Figure 3 and Figure 5 show its $\mathrm{CO}$ signal. For all other galaxies, the spectrum clearly exhibits the signal.

\section{Results and Discussion}

With the CARMA observations, we detected CO in 14 galaxies, 8 of which had not been detected in $\mathrm{CO}$ in the literature, as shown in Table 3. Considering that past rotation curve studies had CO data for only 3 galaxies (NGC 2976, NGC 4605, and NGC 5963; Simon et al. 2005), our data set will significantly increase the sample size of $\mathrm{CO}$ rotation 

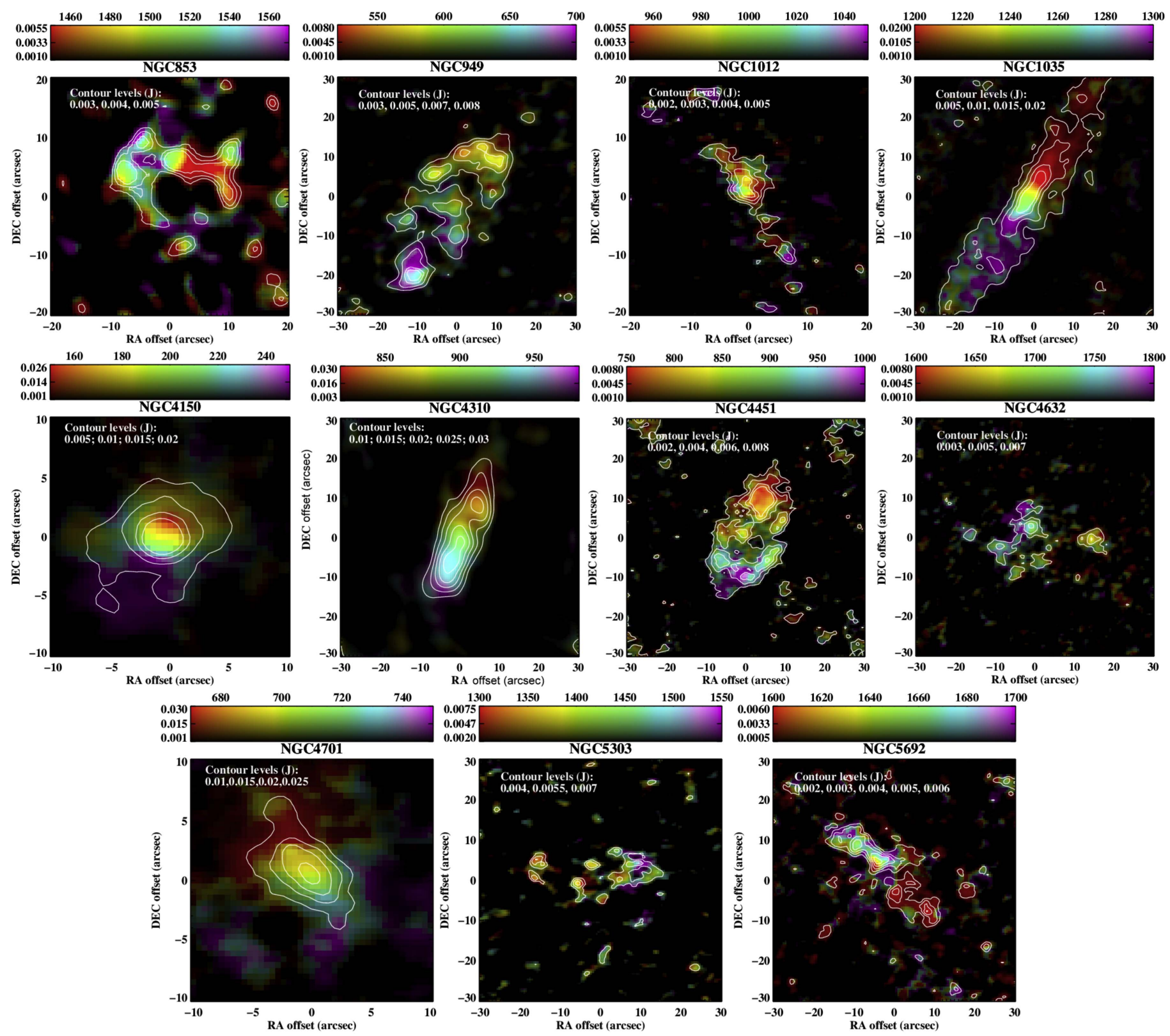

NGC4632
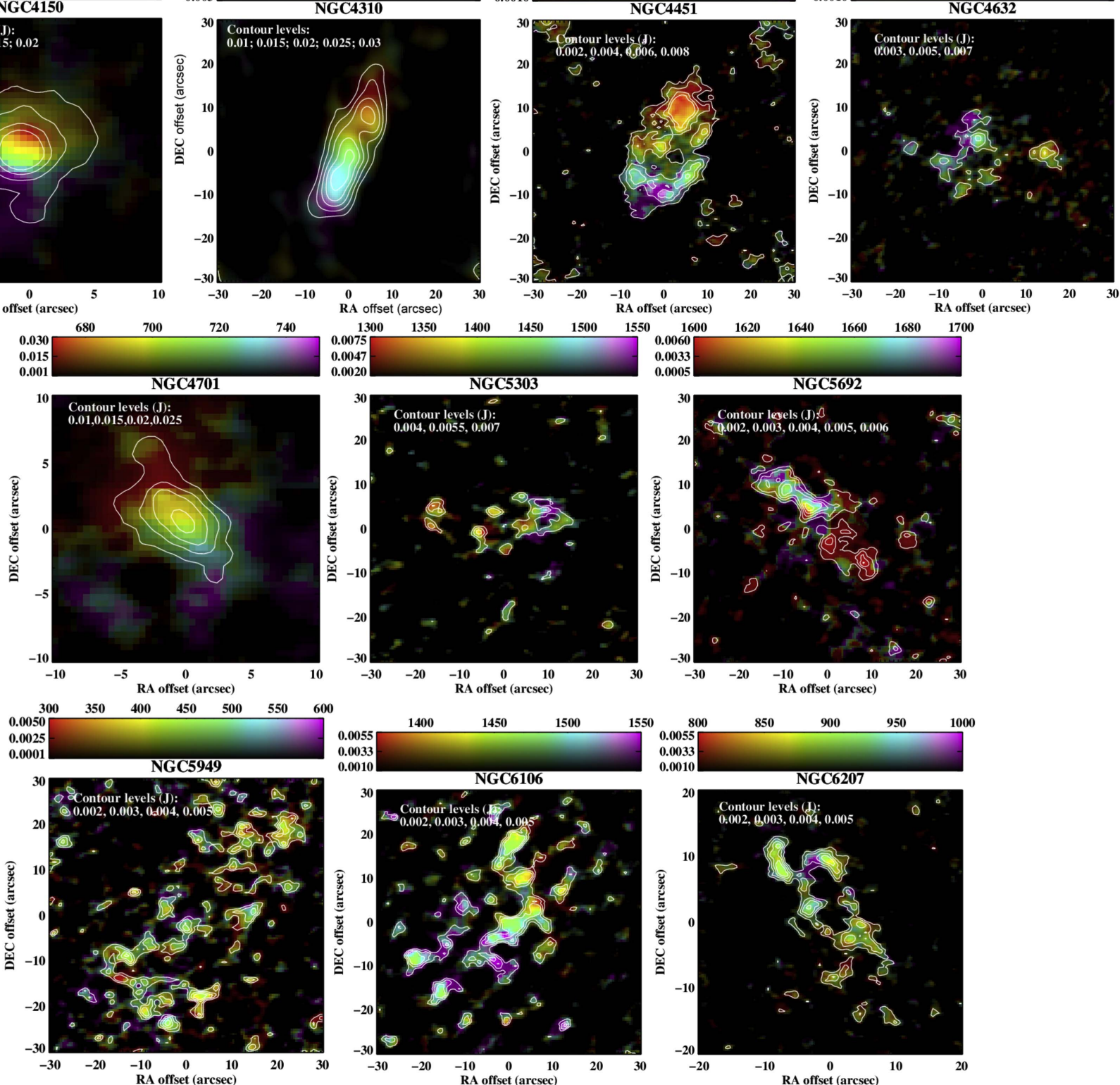

Figure 2. Naturally weighted $\mathrm{CO}(1-0)$ intensity and velocity distribution maps without masking of the CO-detected dwarf galaxies in the sample.

curves that are adequate to accurately measure the dark matter distribution in dwarf galaxies, which is the main goal of our survey. To verify the reliability of our results, we will compare our results with past measurements (Section 5.1) and show that we obtained consistent CO fluxes with Leroy et al. (2005). In addition, we will show that there is a correlation between the 

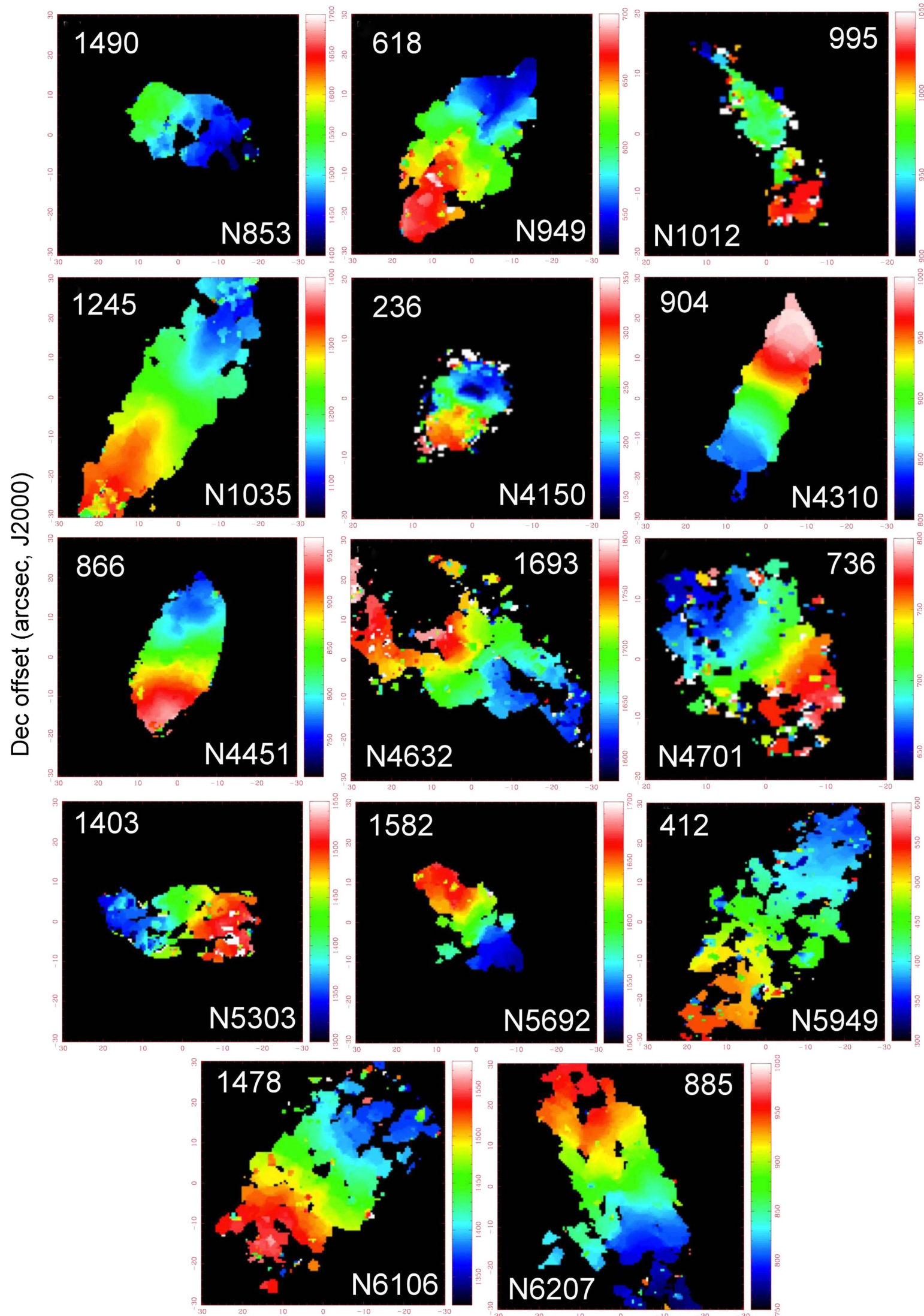

RA offset (arcsec, J2000)

Figure 3. Naturally weighted $\mathrm{CO}(1-0)$ intensity and velocity distribution maps with masking to enhance the SNR. For all galaxies, the R.A. offset is from $+30^{\prime \prime}$ (left) to $-30^{\prime \prime}$ (right), the decl. offset is from $-30^{\prime \prime}$ (bottom) to $+30^{\prime \prime}$ (top); except for NGC 1012 and NGC 4150, whose R.A. and decl. offsets are from $+20^{\prime \prime}$ to $-20^{\prime \prime}$ and $-20^{\prime \prime}$ to $+20^{\prime \prime}$, respectively. The color bar on the right side of each image shows the velocity gradient; the number at the top corner is the systemic velocity. 

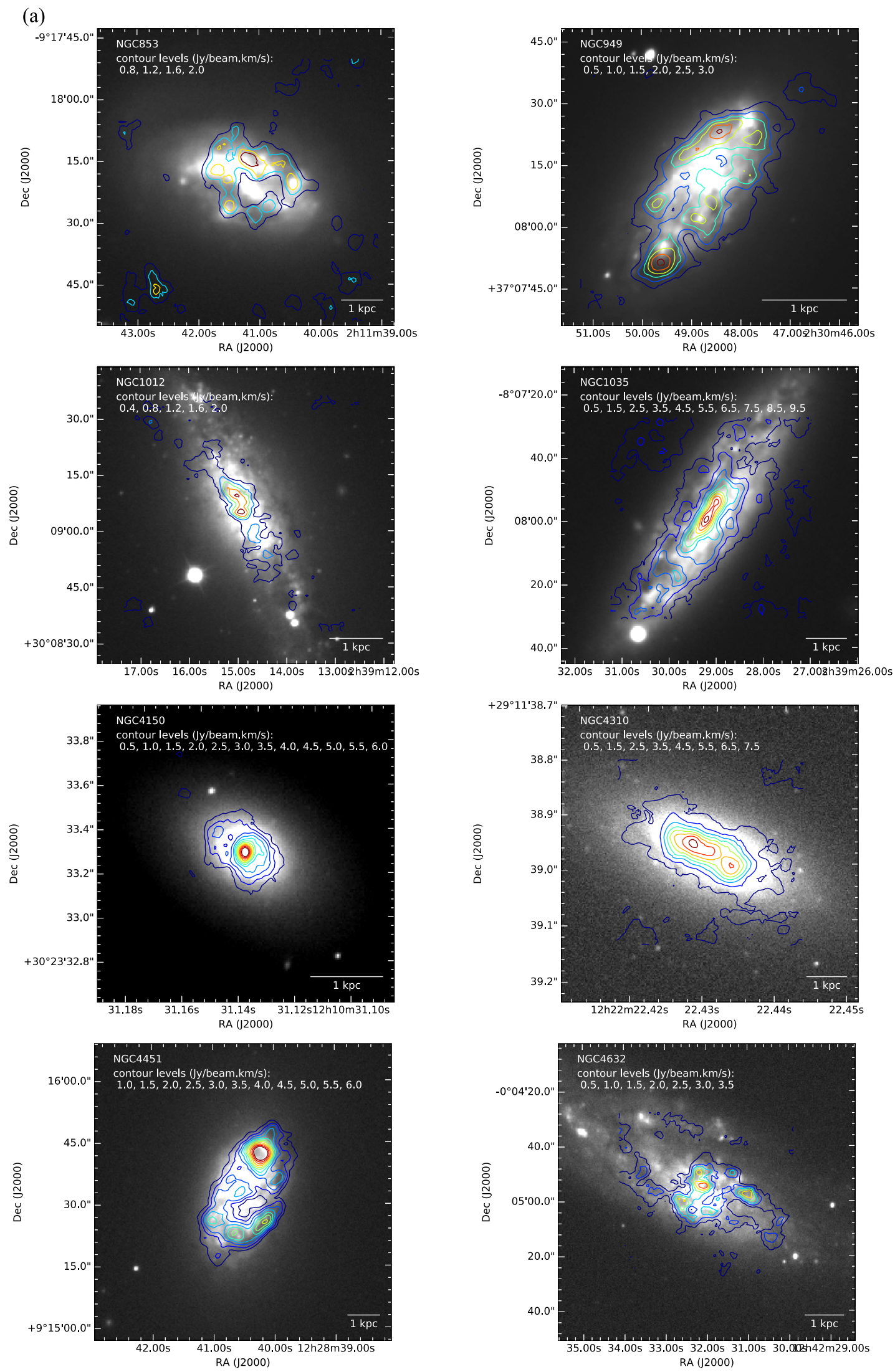

Figure 4. Contours of $\mathrm{CO}(1-0)$ intensity (CARMA data) on $r$-band imaging data, which were taken with SPICAM on the ARC $3.5 \mathrm{~m}$ telescope at Apache Point Observatory. The contour colors correspond to the contour levels listed in the legend for each panel, where the reddest color corresponds to the highest contour level. Exceptions: the $r$-band images of NGC 4150 and NGC 4310 were obtained from the SDSS III archive. 


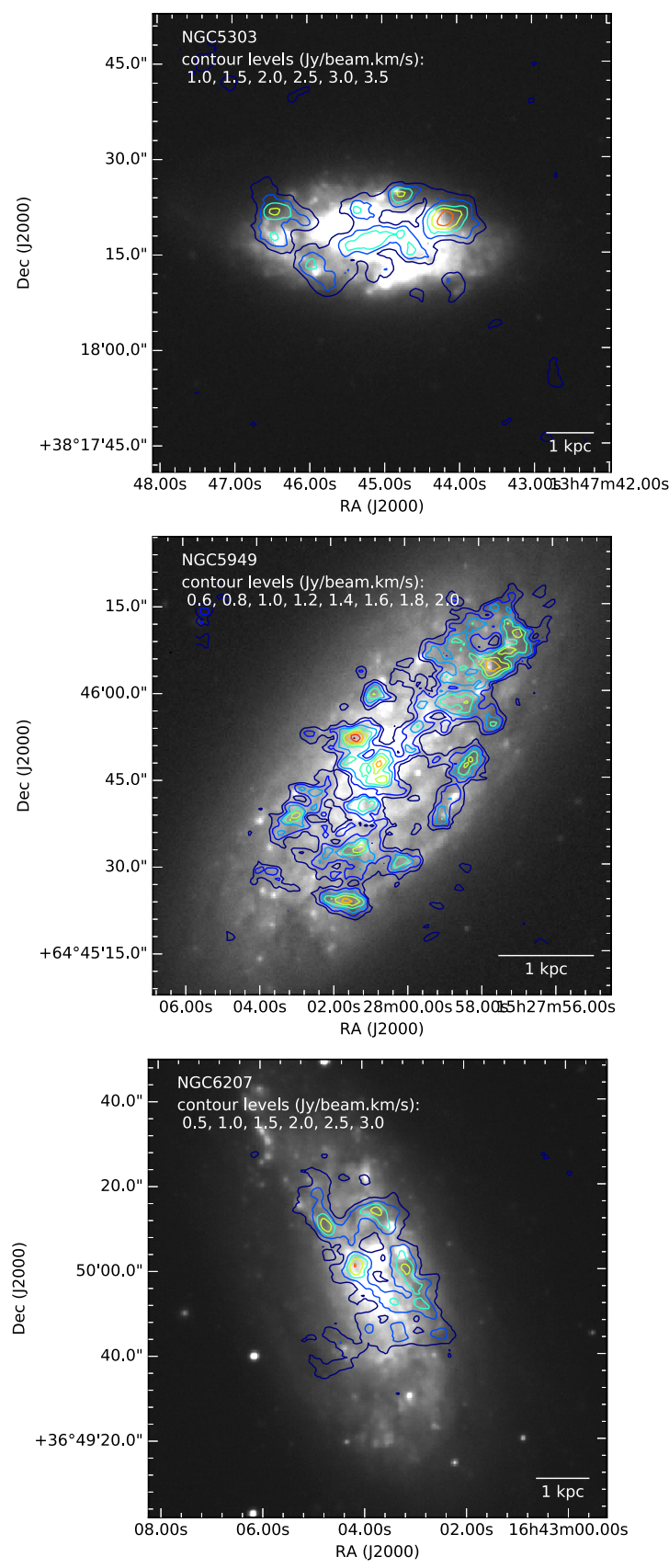

Figure 4. (Continued.)

CO flux and the IR flux, as we expected in our target selection (Section 2).

Table 3 summarizes the survey results for 26 galaxies observed with CARMA, including non-detections. Column (1) lists the galaxy names; column 2 shows the size of the synthesized beam in arcsec; column (3) shows the average rms over the entire image excluding the source emission; column (4a) is the total CO flux density (Jy beam ${ }^{-1} \mathrm{~km} \mathrm{~s}^{-1}$ ) with $1 \sigma$ error bar for the detected targets, or $3 \sigma$ upper limit for the nondetected targets. The error bar was calculated as

$$
\sigma=\operatorname{rms} \times \sqrt{N},
$$

where rms is the average rms and $N$ is the number of synthesized beams per target. Information about the rms value and size of the synthesized beam are determined using the MIRIAD commands IMSTAT and IMLIST.

Columns (4b-8) in Table 3 are intended for comparison between our results and literature results in terms of both $\mathrm{CO}$ measurements and infrared measurements. Column (9) shows the molecular gas content in terms of mass in the CO-detected targets.

\subsection{Flux Comparison}

First, for the single-dish-interferometer CO comparison, column (4b) shows the single-dish-equivalent $\mathrm{CO}$ flux from our observations in $\mathrm{K} \mathrm{km} \mathrm{s}^{-1}$; column (5) shows the integrated $\mathrm{CO}$ flux $\left(I_{\mathrm{CO}}\right)$ from past single-dish observations (Leroy et al. 2005) of our targets where available. The single-dishequivalent $\mathrm{CO}$ flux from our observation in $\mathrm{K} \mathrm{km} \mathrm{s}^{-1}$ was 

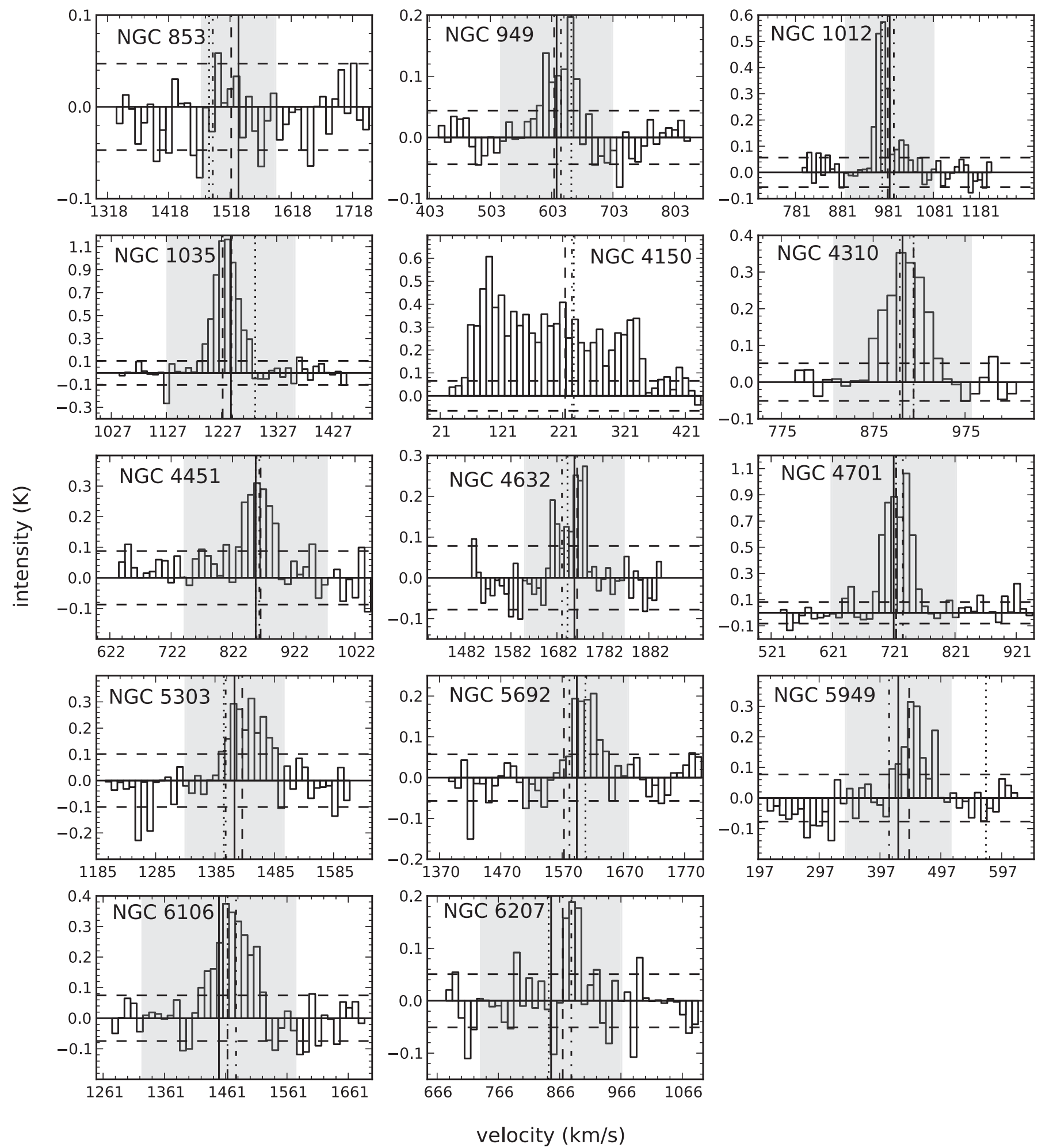

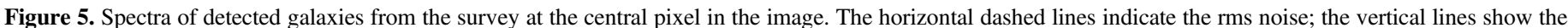

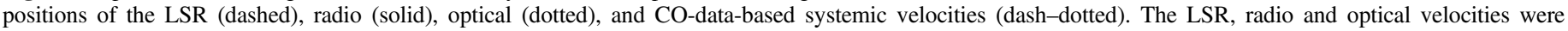
obtained from NED and hyperLEDA. The shaded region shows the $\mathrm{H}$ I line width $W_{20}$.

obtained as

$$
F[\mathrm{~K} \mathrm{~km} / \mathrm{s}]=F[\mathrm{Jy} / \text { beam } \mathrm{km} / \mathrm{s}] \times c_{\mathrm{K} / \mathrm{Jy}} \times \frac{A_{b}}{\pi(55 / 2)^{2}},
$$

where $c_{\mathrm{K} / \mathrm{Jy}}$ is the $\mathrm{K} /\left(\mathrm{Jy} /\right.$ beam) conversion factor, $A_{b}$ is the synthesized beam area (in arcseconds ${ }^{2}$, column (2) in Table 2), and 55 arcsec was the half-power beam width of the Leroy et al. (2005) measurements, which were single-dish observations at $115.27 \mathrm{GHz}$ using the ARO Kitt Peak $12 \mathrm{~m}$ telescope. 

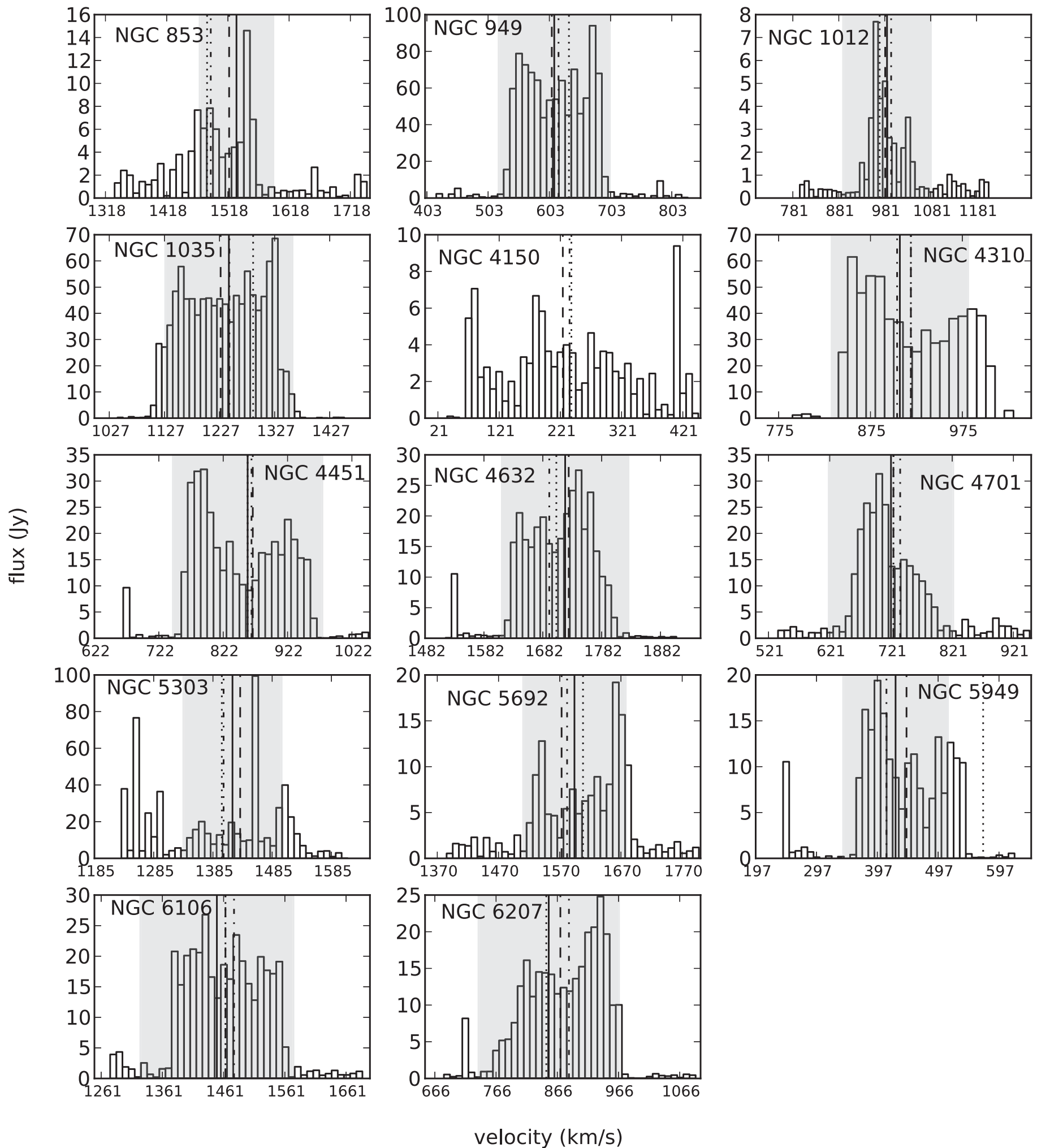

Figure 6. Integrated $\mathrm{CO}$ profile of detected galaxies from the survey. The flux per velocity channel was summed over the entire data cube, where the pixels with intensity $<2.5 \times$ rms were masked. One exception is NGC 5303, whose map is too noisy and the pixels with intensity $<2 \times$ rms were masked because too few pixels had intensity above $2.5 \times$ rms. The vertical lines show the positions of the LSR (dashed), radio (solid), optical (dotted), and CO-data-based systemic velocities (dashdotted). The LSR, radio, and optical velocities were obtained from NED and hyperLEDA. The shaded region shows the H I line width $W_{20}$.

$c_{\mathrm{K} / \mathrm{Jy}}$ and $A_{b}$ were obtained from our moment-0 maps using IMSTAT and IMLIST; $c_{\mathrm{K} / \mathrm{Jy}}$ varies for different targets with a median value of $\sim 8.4$, independently of the clipping levels, whereas $F$ and rms depend on the clipping levels in the maps.
Despite the patchy CO distribution of the targets in Figure 2, for simplicity, we assumed that the entire signal was evenly distributed in the equivalent area of the single-dish beam to calculate the single-dish-equivalent flux. In general, some of 
Table 3

CO Flux of the Galaxies as Measured at CARMA

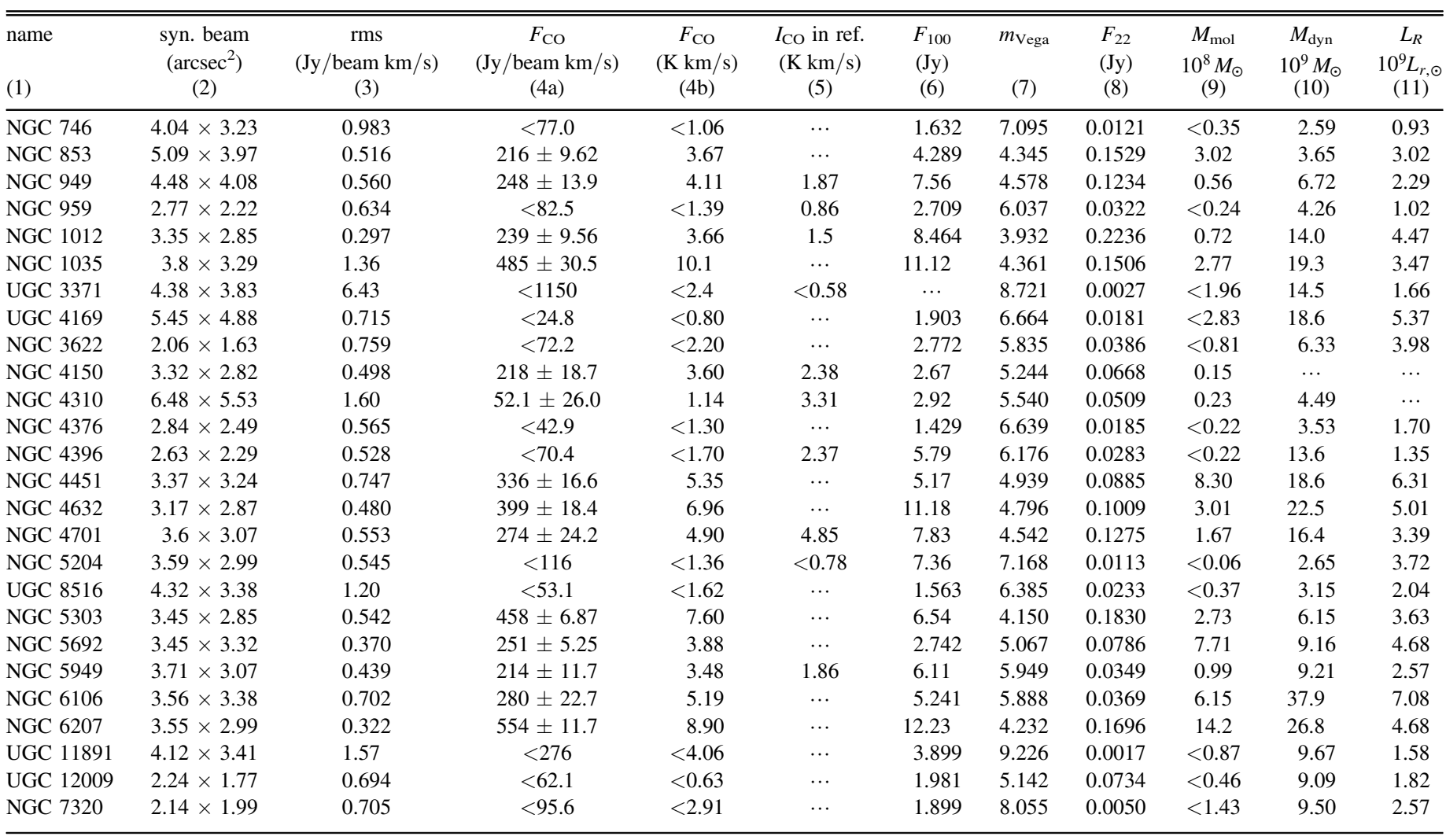

Note. Column (2) is the synthesized beam size. Column (4a) is the CO flux density $\pm 1 \sigma$ (detection) or the limit of detection $(<3 \sigma)$ (non-detection); column (4b) is the equivalent $\mathrm{CO}$ flux density for a single-dish measurement. Column $(5)\left(I_{\mathrm{CO}}\right)$ is the $\mathrm{CO}$ integrated intensity corrected for the main beam efficiency, obtained from Leroy et al. (2005) when data are available. $\mathrm{F}_{100}$ values were obtained from http://ned.ipac.caltech.edu. Column (7) is the Vega magnitude of the galaxies, obtained from the WISE All-Sky Source Catalog for band w4. Column (8) is the 22-micron flux, which was calculated as follows: $F_{22}=8.363 \times 10^{-m \text { vega } / 2.5}$. Column $(9)$ shows the molecular mass of the CO-detected galaxies, which was calculated from the $\mathrm{CO}$ flux in column (4b) assuming the galactic $\mathrm{CO}$-to- $\mathrm{H}_{2}$ conversion factor with the effect of helium. Column (10) shows the dynamical mass, which was calculated from the rotational velocity in Table 1 as $M_{\mathrm{dyn}}=v_{\mathrm{rot}}^{2} R / G M_{\odot} \approx 10^{10} M_{\odot}$. Column $(11)$ shows the $r$-band luminosity, which was obtained from the observations with SPICAM on the ARC $3.5 \mathrm{~m}$ telescope at Apache Point Observatory (N. C. Relatores et al., in preparation). Median values: $M_{\text {mol }} / M_{\text {dyn }} \approx 0.035$ and $\approx 0.010$ for the CO detections and non-detections, respectively; $M_{\text {mol }} / L_{r} \approx 0.078 M_{\odot} / L_{r, \odot}$ and $\approx 0.024 M_{\odot} / L_{r, \odot}$ for the CO detections and non-detections, respectively.

our flux values are $\sim 2$ times larger than Leroy et al.'s values but consistently within the same order of magnitude. This can be explained by our larger primary beam compared to the single-dish primary beam, which would contain more $\mathrm{CO}$ signal (if not the entire $\mathrm{CO}$ signal) of the larger sources.

Second, for the CO-infrared comparison, columns (6) and (7) show the corresponding IRAS 100-micron flux density and WISE w4 (22-micron) Vega magnitude, which we obtained from NED and WISE All-Sky Source Catalog, respectively. We converted the WISE w4 Vega magnitude $\left(m_{\text {Vega }}\right)$ into the 22-micron flux density (in Jansky) using

$$
F_{22}=F_{w 4-0} \times 10^{-m_{\text {Vega }} / 2.5},
$$

where $F_{w 4-0}=8.363 \mathrm{Jy}$ (Jarrett et al. 2011). Jarrett et al. (2011) mentioned that the magnitude-flux conversion for the w4 band may require an additional red-source correction due to a discrepancy between red- and blue-source measurements. However, because our sources do not span a wide range of optical colors, we assumed that such a discrepancy would be uniform among our sources and we therefore did not apply the w4 correction for red sources. The red-source correction, if applied, would only change all values in our list by a constant factor close to unity.

In Figure 7, we plot the 100- and 22-micron flux densities $\left(F_{100}\right.$ and $\left.F_{22}\right)$ of the observed galaxies with their respective $\mathrm{CO}$ flux (or the $3 \sigma$ upper limit for non-detections) from our measurements. In general, the galaxies with high $F_{100}$ are detected in $\mathrm{CO}$ and have high $\mathrm{CO}$ flux, with some exceptions: NGC 4310, NGC 4150, and NGC 5692 (low $F_{100}$ but detected in CO) and NGC 4396, NGC 5204, and UGC 11891 (high $F_{100}$ but undetected in $\mathrm{CO}$ ). We used the function cenken in the R/CRAN package (Helsel 2005; Akritas et al. 1995) to establish the existence of a correlation between $F_{100}$ and $\mathrm{CO}$ flux (including the upper limits) and found that $I_{\mathrm{CO}}=$ $54.09 F_{100}-77.77$ (conversely, $F_{100}=0.01 I_{\mathrm{CO}}+1.49$ (where $I_{\mathrm{CO}}$ is measured in Jy/beam $\mathrm{km} / \mathrm{s}$, and $F_{100}$ in Jy), with a Kendall $\tau$ correlation coefficient of 0.45 (the $p$-value is less than 0.05). Overall, the linear correlation is reasonable because the far-IR region corresponds to the central region of the galaxies with cold molecular clouds.

The correlation between $F_{22}$ and $I_{\mathrm{CO}}$ follows the same trend: the galaxies with high $F_{22}$ generally have high $\mathrm{CO}$ flux, with the only exception of UGC 12009 (high $F_{22}$ but undetected in $\mathrm{CO})$. However, some galaxies with significantly higher mid-IR 

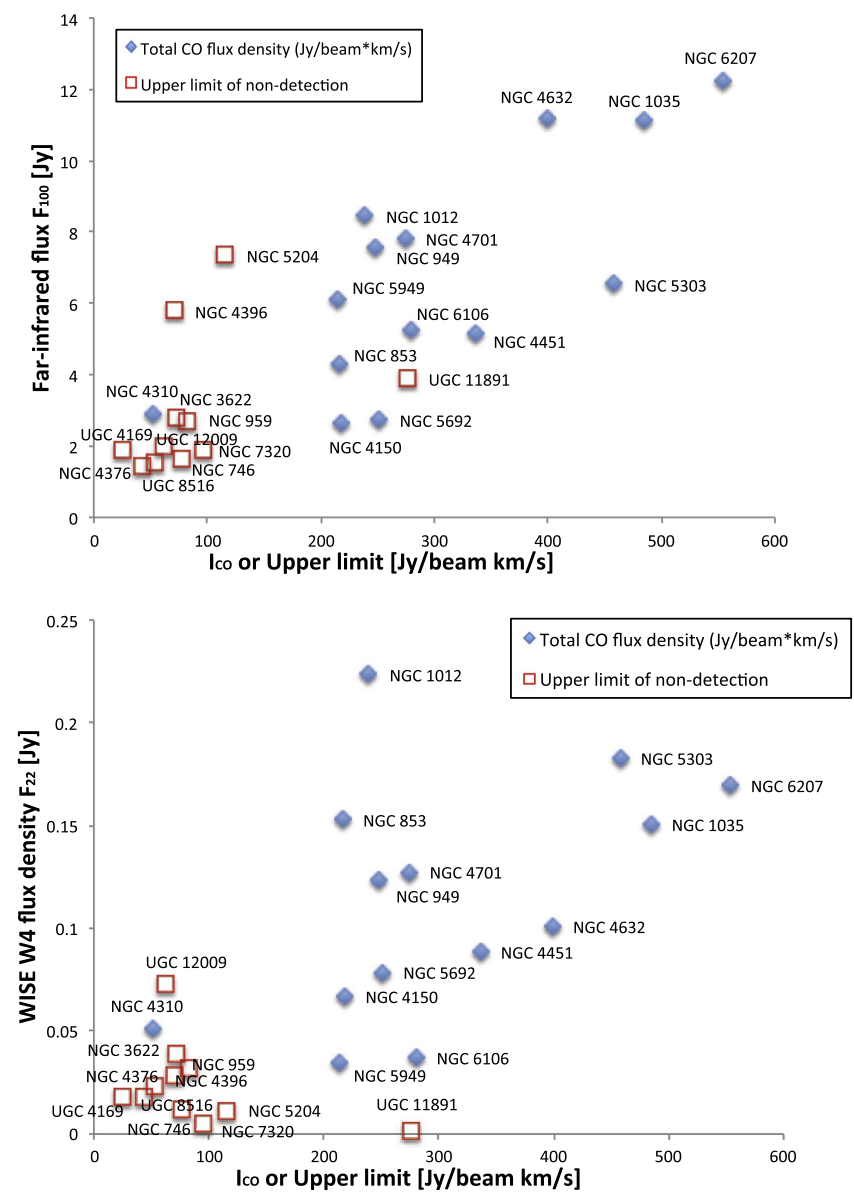

Figure 7. Correlation between the total $\mathrm{CO}$ flux of each target (or $3 \sigma$ upper limit in the case of non-detection) and its corresponding $F_{100 \mu \mathrm{m}}$ and WISE w4 flux density $F_{22} \mu \mathrm{m} . F_{100 \mu \mathrm{m}}$ was obtained from NED and $F_{22} \mu \mathrm{m}$ was converted from the Vega magnitude in band 4, which was obtained from the WISE AllSky source catalog.

flux (compared to the others in the sample) have low $\mathrm{CO}$ flux, such as NGC 853 and NGC 1012, whereas NGC 4310 is detected in $\mathrm{CO}$ despite its small IR flux value. Again, using the function cenken, we found that $I_{\mathrm{CO}}=3.45 \times 10^{3} F_{22}-30.89$ (conversely, $F_{22}=2.50 \times 10^{-4} I_{\mathrm{CO}}+1.59 \times 10^{-2}$ (where $I_{\mathrm{CO}}$ is measured in Jy/beam $\mathrm{km} / \mathrm{s}$, and $F_{22}$ in Jy), with a Kendall $\tau$ correlation coefficient of 0.50 (the p-value is less than 0.05). Thus, compared to the far-IR flux, the mid-IR flux appears to be a better indication of whether a galaxy contains sufficient $\mathrm{CO}$ for detection at our level of sensitivity, but not necessarily a better indication of how much $\mathrm{CO}$ there is in the target. Note that in the original sample selection, we chose our targets partially based on the WISE mid-IR images and not the $F_{100}$ values, so it is not surprising that the correlation between $F_{22}$ and $I_{\mathrm{CO}}$ is better than that between $F_{100}$ and $I_{\mathrm{CO}}$ in our sample. Nonetheless, this correlation could prove to be useful in future studies to help selecting other targets for $\mathrm{CO}$ observations.

\subsection{Molecular Gas Content}

We calculated the mass of molecular gas in the CO-detected targets from the $\mathrm{CO}$ flux, and the results are shown in column (9) of Table 3. Assuming the galactic CO-to- $\mathrm{H}_{2}$ conversion factor $\left[X_{\mathrm{CO}}=2 \times 10^{20} \mathrm{~cm}^{-2}\left(\mathrm{~K} \mathrm{~km} \mathrm{~s}^{-1}\right)^{-1}\right.$; Strong \& Mattox 1996] and following the derivation in Leroy et al. (2005), we have $1 \mathrm{~K} \mathrm{~km} \mathrm{~s}^{-1}$ is equivalent to a molecular surface density of
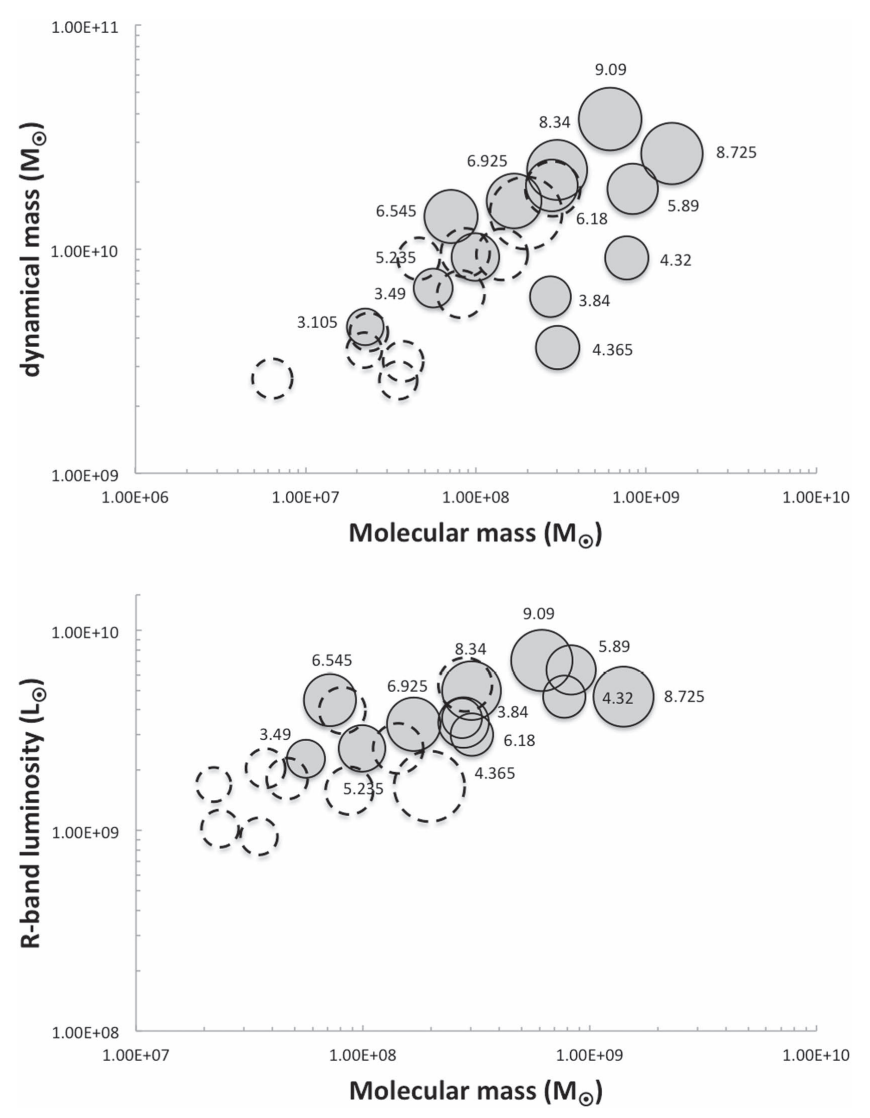

Figure 8. Correlations between the molecular mass, radius, and dynamical mass (top) and $r$-band luminosity (bottom) of the targets in the survey. The COdetected targets are shown with filled circles and the $\mathrm{CO}$ non-detections are shown with empty dashed circles. For the $\mathrm{CO}$ non-detections, the molecular mass was calculated from the $3 \sigma$ upper limit (Table 3 ). The dynamical mass was calculated from the $\mathrm{H}$ I-derived rotational velocity; the $r$-band luminosity was obtained from $r$-band imaging at Apache Point Observatory (N. C. Relatores et al. 2017, in preparation). The size of the bubbles corresponds to the radius in kiloparsecs, whose value is the number shown next to the bubble. To avoid over-cluttering the image, we do not show the numerical values of the radii of the non-detections, which can be found in Table 1.

$\Sigma=4.4 \cos (i) M_{\odot} \mathrm{pc}^{-2}$, which includes both $\mathrm{H}_{2}$ and helium, where $i$ is the inclination of the galaxy. A few dwarfs in our survey have relatively large $M_{\text {mol }}$ (up to $10^{9} M_{\odot}$ ). We note that the Milky Way has a molecular mass on the order of $\approx 10^{9} M_{\odot}$ (Dame 1993). In comparison, the dwarfs in Leroy et al. (2005) had molecular masses on the order of $10^{8}-10^{10} M_{\odot}$. However, the median molecular mass of our galaxies is $\sim 2.75 \times 10^{8} M_{\odot}$, which is consistent with the median molecular mass of dwarf galaxies in Leroy et al. (2005) survey $\left((3 \pm 0.5) \times 10^{8} M_{\odot}\right)$.

We compared the molecular mass with the dynamical mass and $r$-band luminosity, as shown in Figure 8. The dynamical mass was calculated from the $\mathrm{H}$ I-derived rotational velocity $\left(M_{\mathrm{dyn}}\right.$ in Section 2$)$ and the $r$-band luminosity was calculated from $r$-band observations (as mentioned in Section 4, N. C. Relatores et al. 2017, in preparation). In Figure 8, we included both the targets with $\mathrm{CO}$ detection and those without, where the molecular mass of the non-detection was calculated from the $3 \sigma$ upper limits in Table 3 . We also included the radii of the galaxies in the plots (shown as the bubble size).

Figure 8 shows that the galaxies with more dynamical mass and larger size generally contain more molecular gas and that $M_{\text {mol }} / M_{\text {dyn }} \approx 0.035$ for our sample. (For comparison, $M_{\mathrm{mol}} / M_{\mathrm{dyn}} \approx 0.037 \pm 0.007$ for dwarfs in Leroy et al. 2005.) 
However, 3 of the 14 detected galaxies in our sample show large molecular content with correspondingly lower dynamical mass: NGC 853 ( $r=4.36 \mathrm{kpc})$, NGC $5303(r=3.84 \mathrm{kpc})$, and NGC $5692(r=4.32 \mathrm{kpc})$. The CO non-detections are consistent with the same linear correlation between $M_{\mathrm{mol}}$ and $M_{\text {dyn }}$ as the targets with $\mathrm{CO}$ detections and the median upper limit on $M_{\mathrm{mol}} / M_{\mathrm{dyn}}$ is 0.010 for the non-detections. Hence, the achieved depth of the observations was reasonable given expectations for the CO luminosity.

Figure 8 also shows a weak correlation between the $r$-band luminosity and the molecular mass, which implies that larger galaxies with higher stellar mass generally have larger molecular mass, as expected (assuming stellar mass-to-light ratios of $\sim 1$-Adams et al. 2014). Again, NGC 5692 ( $r=4.32 \mathrm{kpc}$ ) has a high $L_{r}$ despite its small size. The median $M_{\mathrm{mol}} / L_{r} \approx 0.078 M_{\odot} / L_{r, \odot}$ for the $\mathrm{CO}$ detections and $\approx 0.024 M_{\odot} / L_{r, \odot}$ for the non-detections. Leroy et al. $2005 \mathrm{did}$ not show any $r$-band correlation, but their ratio of molecular mass and $K$-band luminosity is similar to our $M_{\mathrm{mol}} / L_{r}$, where $M_{\mathrm{mol}} / L_{K} \approx 0.065 \pm 0.008 M_{\odot} / L_{K, \odot}$.

Applying cenken to the values in our sample, we found the correlations

$$
\begin{aligned}
M_{\mathrm{dyn}} & =47.49 M_{\mathrm{mol}}+4.05 \times 10^{9}, \\
L_{r} & =6.77 M_{\mathrm{mol}}+1.78 \times 10^{9}, \\
M_{\mathrm{mol}} & =8.21 \times 10^{7} r-2.68 \times 10^{8},
\end{aligned}
$$

with $\tau=0.42,0.51$ and 0.32 , respectively, where $M_{\text {mol }}$ and $M_{\text {dyn }}$ are measured in $M_{\odot}, L_{r}$ in $L_{\odot}$, and $r$ in kpc. All correlations are statistically significant with $p<0.05$.

There are no resolved $\mathrm{H}$ I data for most of our galaxies, so we cannot make an analogous $M_{\mathrm{mol}}-M_{\mathrm{H}}$ comparison, but considering that our $M_{\mathrm{mol}} / M_{\mathrm{dyn}}$ and $M_{\mathrm{mol}} / L_{r}$ are consistent with past observations, we can assume that $M_{\mathrm{mol}}-M_{\mathrm{H} \text { I }}$ should be $\sim 0.30 \pm 0.05$ (Leroy et al. 2005) for our galaxies. We can apply this value to calculate the atomic gas content for the rotation curves and mass modeling in the second part of our survey (P. N. Truong et al. 2017, in preparation).

\section{Conclusion}

We report the detection of $\mathrm{CO}$ molecular gas in 14 nearby dwarf galaxies, including 6 galaxies that were previously detected in $\mathrm{CO}$ in single-dish measurements. We also present the upper limits for another 12 dwarf galaxies that we did not detect, 2 of which were detected in the published single-dish measurements in the literature, which we believe we did not detect because of weather conditions and lack of sufficient observing time. Most of the detections are late-type spiral galaxies. We compared the CO flux with the mid- and far-IR flux from the WISE catalog and observed a correlation that the mid-IR flux may be a better indication than the far-IR flux of whether a galaxy contains sufficient $\mathrm{CO}$ for detection at the level of instrument sensitivity of CARMA, but not necessarily a better indicator of how much $\mathrm{CO}$ there is in the target. This correlation might prove to be useful in future studies to help choosing other $\mathrm{CO}$ targets for observation. We also observed a weak correlation among the molecular content, dynamical mass, $r$-band luminosity, and size of the galaxies. Using the intensity and velocity maps of the $14 \mathrm{CO}$-detected galaxies in this paper, we will derive the $\mathrm{CO}$ rotation curves in the second paper in the series. In addition, combined with the stellar rotation curves from optical and near-IR imaging data, we will apply mass modeling and infer the dark matter profiles. The full $\mathrm{H} \alpha$ sample will also be provided in future studies, with a comparison between $\mathrm{CO}$ and $\mathrm{H} \alpha$ velocity fields and rotation curves of these galaxies.

We are grateful to Melvyn Wright and Richard Plambeck for their invaluable help with MIRIAD, and to the staff and observers at Owens Valley Radio Observatory (OVRO) and CARMA for their assistance with the observations. Support for CARMA construction was derived from the states of California, Illinois, and Maryland, the James S. McDonnell Foundation, the Gordon and Betty Moore Foundation, the Kenneth T. and Eileen L. Norris Foundation, the University of Chicago, the Associates of the California Institute of Technology, and the National Science Foundation. The CARMA development and operations were supported by the National Science Foundation under a cooperative agreement and by the CARMA partner universities. This publication makes use of data products from the Wide-field Infrared Survey Explorer, which is a joint project of the University of California, Los Angeles, and the Jet Propulsion Laboratory/ California Institute of Technology, funded by the National Aeronautics and Space Administration. This study was partially supported by the NSF grant No. 1140031. A.B. acknowledges support from NSF-AST1412419.

\section{References}

Adams, J. J., Simon, J. D., Fabricius, M. H., et al. 2014, AJ, 789, 63 Akritas, M. G., Murphy, S. A., \& LaValley, M. P. 1995, J. Am. Stat. Assoc., 90,170

Andreani, P., Casoli, F., \& Gerin, M. 1995, A\&A, 300, 43

Arraki, K. S., Klypin, A., More, S., \& Trujillo-Gomez, S. 2014, MNRAS, 438, 1466

Bolatto, A. D., Simon, J. D., Leroy, A., \& Blitz, L. 2002, ApJ, 565, 238

Borriello, A., \& Salucci, P. 2001, MNRAS, 323, 285

Chan, T. K., Keres, D., Onorbe, J., et al. 2015, MNRAS, 454, 2981

Dame, T. M. 1993, in AIP Conf. Proc. 278, Back to the Galaxy, ed. S. Holt \& F. Verter (Melville, NY: AIP), 267

de Blok, W. J. G., \& Bosma, A. 2002, A\&A, 385, 816

de Blok, W. J. G., \& McGaugh, S. S. 1997, MNRAS, 290, 533

de Blok, W. J. G., McGaugh, S. S., Bosma, A., \& Rubin, V. C. 2001a, ApJL, 552, L23

de Blok, W. J. G., McGaugh, S. S., \& Hulst, J. M. 1996, MNRAS, 283, 18 de Blok, W. J. G., McGaugh, S. S., \& Rubin, V. C. 2001b, AJ, 122, 2396 El-Zant, A., Shlosman, I., \& Hoffman, Y. 2001, ApJ, 560, 636

Foot, R. 2016, JCAP, 7, 11

Helsel, D. R. 2005, Nondetects and Data Analysis: Statistics for Censored Environmental Data (New York: Wiley)

Jarrett, T. H., Cohen, M., Masci, F., et al. 2011, ApJ, 735, 112

Kaplinghat, M. 2005, PhRvD, 72, 063510

Karachentsev, I. D., Makarov, D. I., Sharina, M. E., et al. 2003, A\&A, 398, 479

Kato, K., Masao, M., \& Ogiya, G. 2016, in IAU Symp. 317, The General Assembly of Galaxy Halos: Structure, Origin and Evolution, ed. A. Bragaglia et al. (Cambridge: Cambridge Univ. Press), 312

Kuzio de Naray, R., McGaugh, S. S., de Blok, W. J. G., \& Bosma, A. 2006, ApJ, 165, 461

Lees, J. F., Knapp, G. R., Rupen, M. P., \& Phillips, T. G. 1991, ApJ, 379, 177 Leroy, A., Bolatto, A. D., Simon, J. D., \& Blitz, L. 2005, ApJ, 625, 763

Leroy, A. K., Walter, F., Bigiel, F., et al. 2009, AJ, 137, 4670

Mateo, M. L. 1998, ARA\&A, 36, 435

Matthews, L. D., Gao, Y., Uson, J. M., \& Combes, F. 2005, AJ, 129, 1849

Matuszewski, M., Chang, D., Crabill, R. M., et al. 2010, Proc. SPIE, 7735, 77350P McGaugh, S. S., Rubin, V. C., \& de Blok, W. J. G. 2001, AJ, 122, 2381

Navarro, J. F., Frenk, C. S., \& White, S. D. M. 1996, ApJ, 462, 563

Newman, A. B., Treu, T., Ellis, R. S., et al. 2013a, ApJ, 765, 24

Newman, A. B., Treu, T., Ellis, R. S., \& Sand, D. J. 2013b, ApJ, 765, 25

Obreschkow, D., \& Rawlings, S. 2009, MNRAS, 394, 1857

Oh, S.-H., de Blok, W. J. G., Brinks, E., Walter, F., \& Kennicutt, R. C., Jr. 2011, AJ, 141, 193 
Oh, S.-H., de Blok, W. J. G., Walter, F., Brinks, E., \& Kennicutt, R. C., Jr. 2008, AJ, 136, 2761

Oh, S.-H., Hunter, D. A., Brinks, E., et al. 2015, AJ, 149, 180

Ott, J., Stilp, A. M., Warren, S. R., et al. 2012, AJ, 144, 123

Pontzen, A., \& Governato, F. 2012, MNRAS, 421, 3464

Rocha, M., Peter, A. H. G., Bullock, J. S., et al. 2013, MNRAS, 430, 81

Sage, L. J. 1993, A\&A, 100, 537

Sauty, S., Casoli, F., Boselli, A., et al. 2003, A\&A, 411, 381

Simon, J. D., Bolatto, A. D., Leroy, A., \& Blitz, L. 2003, ApJ, 596, 957

Simon, J. D., Bolatto, A. D., Leroy, A., \& Blitz, L. 2005, ApJ, 621, 757

Strong, A. W., \& Mattox, J. R. 1996, A\&A, 308, L21

Swaters, R. A., Madore, B. F., van den Bosch, F. C., \& Balcells, M. 2003a, ApJ, 583, 732
Swaters, R. A., van Albada, T. S., van der Hulst, J. M., \& Sancisi, R. 2002, A\&A, 390, 829

Swaters, R. A., Verheijen, M. A. W., Bershady, M. A., \& Andersen, D. R. 2003b, ApJL, 587, L19

Tonini, C., Lapi, A., \& Salucci, P. 2006, ApJ, 649, 591

van den Bosch, F. C., \& Swaters, R. A. 2001, MNRAS, 325, 1017

Walter, F., Brinks, E., de Blok, W. J. G., et al. 2008, AJ, 136, 2563

Weinberg, M. D., \& Katz, N. 2007a, MNRAS, 375, 425

Weinberg, M. D., \& Katz, N. 2007b, MNRAS, 375, 460

Welch, G. A., \& Sage, L. J. 2003, ApJ, 584, 260

Wright, E. L., Eisenhardt, P. R. M., Mainzer, A. K., et al. 2010, AJ, 140, 1868

Young, J. S., Xie, S., Tacconi, L., et al. 1995, ApJ, 98, 219 